\title{
O profissional educador social no cotidiano de adolescentes de unidade de acolhimento institucional: uma revisão integrativa
}

The professional social educator in the daily life of adolescents of reception unit: an integrative review

El educador social profesional en la vida cotidiana de los adolescentes de la unidad de recepción: una revisión integrativa

Lucia Helena Garcia Penna

ORCID: https://orcid.org/0000-0001-9227-628X Universidade do Estado do Rio de Janeiro, Brasil E-mail: luciapenna@terra.com.br

Claudia Rosane Guedes

ORCID: https://orcid.org/0000-0002-1926-0756

Universidade do Estado do Rio de Janeiro, Brasil

E-mail: docente.cg@gmail.com

Rosemary Ribeiro

ORCID: https://orcid.org/0000-0003-2119-5452

Secretaria Municipal de Saúde/Caxias, Brasil

E-mail: rosemaryrib@gmail.com

Adriana Lenho de Figueiredo Pereira

ORCID: https://orcid.org/0000-0002-2563-6174

Universidade do Estado do Rio de Janeiro, Brasil

E-mail: adrianalenho.uerj@gmail.com

Liana Viana Ribeiro

ORCID: https://orcid.org/0000-0001-5566-2974

Universidade do Estado do Rio de Janeiro, Brasil

E-mail: liana.uerj@gmail.com

Joana Iabrudi Carinhanha

ORCID: https://orcid.org/0000-0001-9301-7327

Universidade do Estado do Rio de Janeiro, Brasil

E-mail: joanaiabrudi@gmail.com

Michele Marim da Silva

ORCID: https://orcid.org/0000-0002-0768-6717

Universidade do Estado do Rio de Janeiro, Brasil

E-mail: michelemarimds@gmail.com

Vanessa dos Santos Pereira

ORCID: https://orcid.org/0000-0002-1089-5536

Universidade do Estado do Rio de Janeiro, Brasil

E-mail: vanessa.sotnas.pereira@gmail.com

Susane Vasconcelos

ORCID: https://orcid.org/0000-0001-8819-3385

Universidade do Estado do Rio de Janeiro, Brasil

E-mail: susanevasconcelos@hotmail.com

Selma Villas Boas Teixeira

ORCID: https://orcid.org/0000-0001-8799-0243

E-mail: selma.teixeira@unirio.br

\section{Resumo}

Objetivou analisar o papel do educador social junto aos adolescentes nas unidades de acolhimento institucional. Pesquisa qualitativa, descritiva do tipo integrativa, realizada na Biblioteca Virtual em Saúde, com publicações da última década, associando as palavras chaves e os descritores em saúde: abrigo; educador social; educadores e unidade de acolhimento. Os resultados apontaram a relevância do papel do educador social na atualidade. O perfil dos educadores é majoritariamente feminino; em geral encontram-se na faixa etária de 20 a 66 anos, e com ensino médio. Exercem educação não formal que propõe o desenvolvimento do ser social, desenvolvida em grupos em situação de risco social no contexto das unidades de acolhimento, nem sempre obtendo capacitação específica para atuação. Estabelecem o cuidado, a partir de sua própria experiência pessoal. Considera-se que esta profissão favoreça a emancipação e transformação dos sujeitos que se encontram 
em situação de risco e vulnerabilidade social, sendo figura referência nas unidades de acolhimento institucional de adolescentes. Sua atuação tem sido essencial na educação não formal dos acolhidos, compartilhando saberes em forma de diálogo.

Palavras-chave: Abrigo; Educador social; Unidade de acolhimento.

\begin{abstract}
This study aimed to analyze the role of social educators with adolescents in institutional reception units. Qualitative, descriptive integrative research, carried out in the Virtual Health Library, with publications of the last decade, associating the Keys Word and the descriptors in health: shelter; social educator; educators and host unit. The results pointed out the relevance of the role of social educators today. The profile of educators is mostly female; are generally between 20 and 66 years of age and with high school education. They exercise non-formal education that proposes the development of the social being, developed in groups at social risk in the context of the host units, not always obtaining specific training for action. They establish care, based on their own personal experience. It is considered that this profession favors the emancipation and transformation of subjects who are in a situation of risk and social vulnerability, being a reference in the institutional reception units of adolescents. Its performance has been essential in the non-formal education of the welcomed, sharing knowledge in the form of dialogue.
\end{abstract}

Keywords: Shelter; Social educator; Reception unit.

\title{
Resumen
}

Este estudio tenía como objetivo analizar el papel de los educadores sociales con adolescentes en las unidades de acogida institucional. Investigación cualitativa y descriptiva del tipo integrador, llevada a cabo en la Biblioteca Virtual de Salud, con publicaciones de la última década, asociando las palabras clave y los descriptores en salud: refugio; educador social; educadores y unidad de acogida. Los resultados señalaron la relevancia del papel de los educadores sociales hoy en día. El perfil de los educadores es en su mayoría femenino; generalmente tienen entre 20 y 66 años de edad y con educación secundaria. Ejercen una educación no formal que propone el desarrollo del ser social, desarrollado en grupos de riesgo social en el contexto de las unidades de acogida, no siempre obteniendo formación específica para la acción. Establecen el cuidado, basado en su propia experiencia personal. Se considera que esta profesión favorece la emancipación y transformación de sujetos que se encuentran en situación de riesgo y vulnerabilidad social, siendo un referente en las unidades de acogida institucional de adolescentes. Su desempeño ha sido esencial en la educación no formal de los acogidos, compartiendo conocimientos en forma de diálogo.

Palabras clave: Refugio; Educador social; Unidad de acogida.

\section{Introdução}

As unidades de acolhimento institucional são serviços especializados em proteção social especial considerado de alta complexidade, para crianças e adolescentes, afastados temporariamente do seu núcleo familiar e/ou comunitário, bem como os que se encontram em situação de abandono, ameaça ou violação de direitos. Essas unidades integram o Sistema Único de Assistência Social - SUAS (Brasil, 1990, Brasil, 2009, Souza et al., 2014, Bassoli \& Figueredo, 2020, Ito \& Azevêdo, 2021).

O acolhimento institucional é considerado medida provisória e excepcional, utilizável como forma de transição para reintegração familiar ou, não sendo esta possível, para colocação em família substituta, não implicando privação de liberdade, conforme prevê a Lei no 12.010, de 2009 (Brasil, 2009) São unidades que possui característica de assistência psicossocial, assistida por equipe multiprofissional composta por psicólogo, pedagogo, assistente social e o educador social (ES) (Souza et al., 2014, Bassoli \& Figueredo, 2020).

Dentre os profissionais que assistem a população de crianças e adolescentes acolhidas encontra-se o Educador Social (ES). Este tem sido figura fundamental no cotidiano de adolescentes em situação de acolhimento, sendo-lhes a referência mais próxima de um adulto. Nas unidades de acolhimento, o jovem geralmente, busca construí vínculos com pessoas tidas como "modelos de identificação", profissionais referências para os jovens nas unidades de acolhimento (Brasil, 2009, Souza et al, 2014). O ES define-se como tal, quando se dirige à educação que propõe o desenvolvimento do ser social, à educação desenvolvida em grupos em situação de risco social e, por fim, por meio de educação não formal (Lucca et al., 2018, Bassoli, 2020, Trilla, 2003). 
O ES desenvolve ações afirmativas, mediadoras, formativas, educativas com diversas populações vulneráveis, em distintos âmbitos institucionais, comunitários e sociais, em programas e projetos socioeducativos, a partir das políticas públicas definidas pelas esferas públicas (Brasil, 2015, Lucca et al., 2018, Souza et al., 2014). A função do ES nas unidades de acolhimento institucional é acompanhar o cotidiano do adolescente; atuar na prestação de auxílio e apoio aos acolhidos, bem como organização do ambiente de acolhimento, assegurar a alimentação, higiene e o acompanhamento nos serviços a eles prestados (de saúde, educação, entre outros). O ES pode auxiliar o adolescente a lidar com sua história de vida e contribuir para o fortalecimento da autoestima e construção identitária, assim como apoiá-lo no processo de desligamento para a reinserção familiar (Ito \& Azevêdo, 2021, Avoglia et al., 2012. Elage et al., 2011).

Frequentemente, tais profissionais, ao desempenharem funções cotidianas relacionadas aos adolescentes, acabam estabelecendo vínculos afetivos e proporcionam oportunidades de reflexão conjunta que os impulsionam a aprender e pensar, usando a própria vivência. As ações dos ES visam a resgatar tanto a confiança do adolescente acolhido, quanto a sua capacidade de enfrentar os desafios que, certamente, encontrarão no processo de reintegração social (Brasil, 2009, Elage et al., 2011).

Considerando a longa permanência dos adolescentes na instituição, estes em geral, recorrem ao ES para minimizar suas dúvidas, dividir conflitos e ansiedades. Dessa forma, estes contribuem com sua referência de vida junto às questões de saúde. Esse convívio diário reforça os laços, criando-se um vínculo. Esses profissionais, em geral, são pessoas com mais vivências, com uma história de vida pregressa, favorecendo diálogos de saberes e ideias (Elage et al., 2011, Oliveira, 2017; Cavalcante et al., 2012, Corrêa et al., 2013).

Para além das atividades que visam à reinserção social dos adolescentes, está a condição de referência de vida, de promoção da saúde desses educadores junto aos acolhidos. Entretanto, muitos deles baseiam-se em suas experiências de vida, atuam de forma empírica, promovendo, por conseguinte, ações repletas de preconceitos e valores na promoção à saúde dos acolhidos (Barros \& Naiff, 2015, Cavalcante et al., 2012, Corrêa et al., 2013).

O perfil dos ES, majoritariamente, é de mulheres que estabelecem o cuidado a partir de sua própria condição feminina. Possui o ensino médio, idade de 20 a 66 anos, encontra-se em período central de seu desenvolvimento individual, tanto na esfera familiar, como nas experiências do casamento e da maternidade, em grande parte ainda no período reprodutivo ou com filhos na faixa etária dos adolescentes acolhidos, o que constitui fator positivo para o processo de trabalho (Cavalcante et al., 2012; Corrêa et al., 2013; Elage et al., 2011; Nörnberg \& Menezes, 2011).

Comumente, faz-se necessário que esses profissionais se identifiquem em suas ações cotidianas, de forma a compreenderem o impacto de suas ações para o desenvolvimento desses adolescentes. Entretanto, associando tais ações ao perfil dos ES é possível afirmar que as maneiras de cuidar e de orientar esses adolescentes são permeadas por suas habilidades, afinidades, valores e vivências pessoais, determinando sua abordagem junto aos adolescentes. Além disso, é necessário destacar que as educadoras são mulheres que vivem em um contexto social com desigualdades de gênero, onde o olhar sobre o papel desempenhado tem influência de uma sociedade ainda patriarcal, trazendo consequência sobre sua promoção da saúde (Cunha, 2011; Elage et al., 2011; Cavalcante et al., 2012; Corrêa et al., 2013). Considerando a frágil visibilidade do papel do ES, mas destacando sua importante referência no cuidado de adolescentes acolhidos objetiva-se, a partir de uma pesquisa bibliográfica, analisar o papel desempenhado pelo ES junto aos adolescentes em unidades de acolhimento.

\section{Metodologia}

Trata-se de uma pesquisa qualitativa do tipo de revisão integrativa. Esse tipo de pesquisa que possibilita a síntese dos estudos publicados sobre determinado tema, obtendo, a partir deles, conclusões que irão contribuir com as diversas tomadas de 
decisão (Pereira, Shitsuka, Parreira \& Shitsuka, 2018), (Carvalho, Ferreira \& Ferreira, 2020). Esse tipo de estudo permite uma aproximação da problemática a ser estudada (Botelho et al., 2011). A coleta foi elaborada segundo o fluxograma contido no Preferred Reporting Items for Systematic reviews and Meta-Analyses (PRISMA), a qual utiliza uma lista de verificação aplicada no decorrer da revisão, com o intuito de aprimorar os relatos obtidos (Liberati et al., 2009).

Utilizou-se a Biblioteca Virtual em Saúde (BVS) que abarca uma gama de bases de dados, como: Medical Literature Analysis and Retrieval System Online (MEDLINE), Literatura Latino-Americana e do Caribe em Ciências da Saúde (LILACS), Banco de Dados em Odontologia (BBO) e Index Psicologia. Este espaço é caracterizado a partir da integração de fontes de informação em saúde, promovendo a democratização e a ampliação do acesso à informação científica e técnica em saúde na América Latina e Caribe.

Para a operacionalização da presente revisão integrativa foram consideradas seis etapas: identificação do tema e seleção da questão norteadora; estabelecimento dos critérios de inclusão e exclusão; seleção dos artigos; categorização dos artigos; análise dos estudos; e síntese do conhecimento (Carvalho, Ferreira \& Ferreira, 2020). O marco temporal (2009 a 2018) foi estabelecido ao considerar a edição do documento Orientações Técnico sobre os Serviços de Acolhimento para Crianças e Adolescentes, elaborado pelo Ministério de Desenvolvimento Social (Brasil, 2009), que estabelece os parâmetros para o funcionamento, em suas diferentes modalidades de oferta, bem como as orientações metodológicas para o manejo de crianças e adolescentes nos espaços de acolhimento no país.

Para conduzir a revisão integrativa, formulou-se a seguinte questão norteadora: "Qual o papel do educador social no cotidiano de adolescentes de unidade de acolhimento?”. Após realizar a busca no rol de Descritores em Ciências da Saúde (DeCS), não se identificou o termo controlado para "Educador Social". Optou-se por utilizar as palavras genéricas "educador social" e "unidade de acolhimento", conexas aos descritores: "abrigo"; "educadores" associados ao booleano AND foi possível estabelecer estratégias de buscas nas bases de dados. (Vide Figura 1).

O levantamento bibliográfico potencial foi constituído de 45 trabalhos científicos. O processo de seleção das produções elencadas a partir dos descritores e/ou palavras-chaves com o refinamento a partir dos critérios de inclusão e exclusão. Estabelece-se como critérios de inclusão: textos completos e disponíveis na Web, no formato de artigo e produções nacionais. E excluíram-se teses, dissertações, monografias, documentos governamentais, resenhas, artigos em duplicidade e que não atendiam aos objetivos deste estudo. Após esta etapa perfizeram um total de 34 artigos. Numa segunda avaliação, selecionou-se os temas pertinentes aos objetivos do trabalho, destacando-se 11 artigos para análise. As produções foram organizadas por título, autor e ano de publicação, local de origem do artigo, área do conhecimento e metodologia.

As produções foram analisadas qualitativamente, quanto as suas ideias principais, utilizando a técnica de Análise de Conteúdo (Bardin, 2016). Emergiram duas categorias: 1) O processo de formação do educador social, e a categoria 2) "A figura do educador social como um agente de transformação na vida de adolescentes acolhidos" 
Figura 1 - Fluxograma explicativo da estratégia de busca e seleção dos estudos, Rio de janeiro, 2020.

\section{BASES DE DADOS:}

LILACS, Index Psicologia - Periódicos técnico-científicos, MEDLINE, BDENF - Enfermagem

\section{BUSCA}

\section{DESCRITORES}

Abrigo AND educadores AND (db:("LILACS" unidade de acolhimento AND educadores AND (db:("LILACS" OR "INDEXPSI")) OR "INDEXPSI" OR "MEDLINE" OR "BDENF")) unidade de acolhimento AND educadores AND (db:("LILACS" OR "INDEXPSI")) abrigo AND educador social AND (db:("LILACS" OR "INDEXPSI" OR "MEDLINE" OR "tese"))

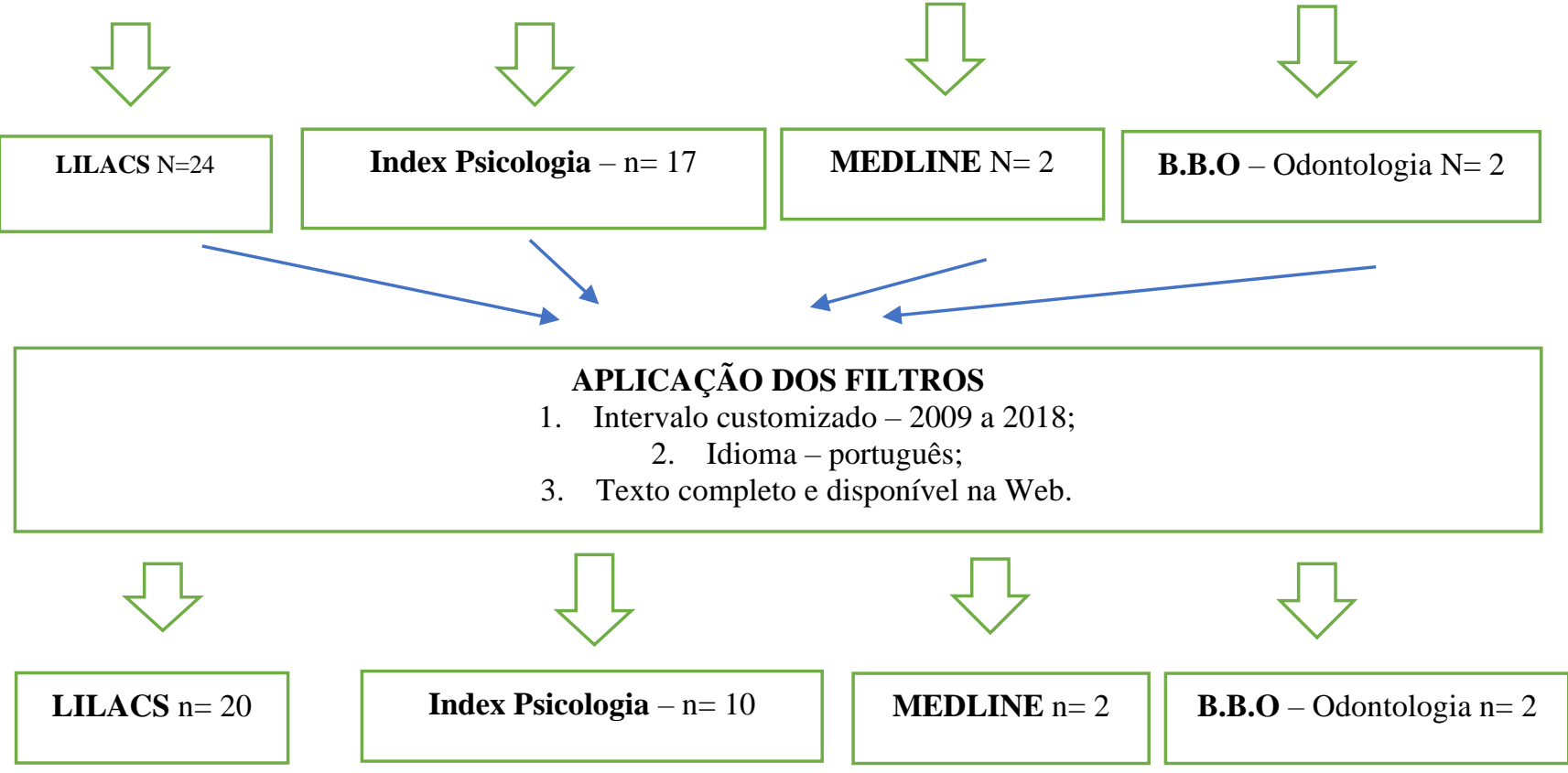

\section{Leitura dos títulos e resumos $-\mathrm{n}=34$}

Aplicação dos critérios - inclusão /exclusão/duplicidade

Total $\mathrm{n}=23$

Amostra final da revisão $\mathrm{n}=11$

Fonte: Autores (2020). 
Os dados selecionados foram organizados num quadro: ano, título e autores, local de origem, área do conhecimento e metodologia (Quadro 1).

Quadro 1 - Caracterização dos artigos selecionados para compor a amostra final da revisão, Rio de Janeiro 2020.

\begin{tabular}{|c|c|c|c|}
\hline Título e autores & $\begin{array}{l}\text { Local } \\
\text { de } \\
\text { origem }\end{array}$ & $\begin{array}{c}\text { Área } \\
\text { conhecimento }\end{array}$ & Metodologia \\
\hline $\begin{array}{l}\text { Capacitação para educadores de abrigo de crianças e } \\
\text { adolescentes: identificando representações sociais. } \\
\text { Barros \& Naiff, (2015) }\end{array}$ & RJ & Psicologia (PSI) & Qualitativa \\
\hline $\begin{array}{l}\text { Conhecimento dos educadores do abrigo Tereza de Jesus } \\
\text { sobre saúde bucal infantil. } \\
\text { Bispo } \text { et al., (2015). }\end{array}$ & SP & Odontologia & Quantitativa \\
\hline $\begin{array}{l}\text { Formação de educadores sociais: Profissionalização Técnica, } \\
\text { Para Quê? } \\
\text { Pereira, Antônio, (2015). }\end{array}$ & BA & Educação & Qualitativa \\
\hline $\begin{array}{l}\text { Escuta afetiva: possibilidades de uso em contextos de } \\
\text { acolhimento infantil. } \\
\text { Trivellato } \text { et al., (2013). }\end{array}$ & SP & PSI & Qualitativa \\
\hline $\begin{array}{l}\text { Educadores de abrigo: concepções sobre desenvolvimento e } \\
\text { práticas de cuidado em situação de brincadeira. } \\
\text { Correa \& Cavalcante, (2013). }\end{array}$ & SP & PSI & Qualitativa \\
\hline $\begin{array}{l}\text { Concepção de profissionais de unidades de acolhimento sobre } \\
\text { a maternidade em adolescentes abrigadas. } \\
\text { Penna } \text { et al., (2012). }\end{array}$ & RJ & Enfermagem & Qualitativa \\
\hline $\begin{array}{l}\text { Educador Social: imagem e relações com crianças em situação } \\
\text { de acolhimento institucional. } \\
\text { Avoglia } \text { et al., (2012). }\end{array}$ & $\mathrm{CE}$ & PSI & Qualitativa \\
\hline $\begin{array}{l}\text { Percepção de educadores de abrigo: o seu trabalho e a criança } \\
\text { institucionalizada. } \\
\text { Magalhães et al., (2012). }\end{array}$ & SP & PSI & Qualitativa \\
\hline $\begin{array}{l}\text { Qualidade de vida dos educadores sociais em abrigos de } \\
\text { proteção a crianças e adolescentes. Brito \& Souza (2011). }\end{array}$ & MS & PSI & Quantitativa \\
\hline $\begin{array}{l}\text { Holding e Rêverie: postura do coordenador de grupo de } \\
\text { reflexão com educadoras em um abrigo. Lam (2010). }\end{array}$ & SP & PSI & Qualitativa \\
\hline $\begin{array}{l}\text { Violência vivenciada pelas adolescentes em situação de rua na } \\
\text { ótica dos profissionais cuidadores do abrigo. } \\
\text { Penna } \text { et al., (2010). }\end{array}$ & RJ & Enfermagem & Qualitativa \\
\hline
\end{tabular}

Fonte: Autores (2020).

\section{Resultados}

Quanto à recorrência anual das publicações observam-se lacunas nos últimos três anos e nos anos de 2009 e 2014 . Em relação aos onze textos encontrados, nota-se uma distribuição de artigos variando entre duas e três publicações por ano, sendo três artigos no ano de 2015, seguida por uma distribuição uniforme de dois artigos nos anos de 2013, 2012, 2011 e 2010.

A região Sudeste destaca-se como a região que produziu mais estudos, com sete publicações, seguida da Norte, com três, e da Centro-Oeste, com uma, não havendo produções nas regiões Norte e Sul. Em relação às regiões originárias dos artigos, a Sudeste tem a maior proporção observada: cinco artigos de São Paulo (Pereira, 2015, Trivellato et al., 2013, Corrêa \& Cavalcante, 2013, Magalhães et al., 2011, Lam, 2010) três do Rio de Janeiro (Barros \& Naiff, 2015, Penna et al.,2012; 
Penna et al., 2010), um artigo da Bahia (Pereira, 2015), um artigo do Ceará (Avoglia et al., 2012) e um artigo de Mato Grosso do Sul (Brito \& Souza, 2011).

Acredita-se que esse fato se baseia na maior concentração de unidades de acolhimento no Sudeste, consequência do número de crianças/adolescentes com ameaça ou violação de direitos. O Censo SUAS, de 2017, aponta totalidade 5.589 Unidades de Acolhimento Institucional, sendo a concentração maior no Sudeste, com 2.993 e a menor, no Norte, com 222 (Brasil, 2018).

Referindo-se à área de conhecimento, nas Ciências Humanas a Psicologia alcança 80,2\% das produções. Além disso, a inserção do psicólogo no atendimento nas unidades de acolhimento afina-se com a aprovação das Orientações Técnicas para os Serviços de Acolhimento e com a Nova Lei da Adoção (Lei no 12.010, de 3 de agosto de 2009), que reforça a importância e o papel da equipe técnica na interlocução entre os serviços, as organizações e as instituições na busca por uma rede de proteção social a esse grupo populacional (Brasil, 2009). Nas demais estudadas, identificaram-se áreas de atuação das ciências da saúde, como a Enfermagem e a Odontologia versando questões da promoção da saúde.

As características das produções constatam-se uma escassez, sobretudo recentemente, de produções sobre o ES de unidades de acolhimento institucional de crianças/adolescentes. Vale mencionar a visibilidade da temática nas Ciências Humanas, predominando a Psicologia. Isso se deve à participação dessa categoria na equipe de referência dos serviços de acolhimento, conforme definido pela NOB/RH-SUAS (Brasil, 2006, Corrêa et al., 2013).

Ainda se observam produções nas Ciências Humanas no serviço de Psicologia (Barros \& Naiff, 2015; Trivellato et al., 2013, Correa et al., 2013, Avoglia et al., 2012, Magalhães et al., 2011, Brito \& Souza, 2011, categoria também integrante da equipe técnica das unidades, uma produção na Educação (Pereira, 2015). Nas Ciências da Saúde, a Enfermagem (Penna et al., 2012; Penna et al., 2010) e a Odontologia (Bispo et al.,) abordando a promoção e o cuidado à saúde.

Em relação à abordagem metodológica, observa-se a predominância de pesquisas qualitativas, em 80,2 \% das produções. Neste grupo, observaram-se pesquisas do tipo descritivo (Teoria das Representações Sociais), observações da rotina institucional, utilização do Inventário de Conhecimento do Desenvolvimento Infantil (KIDI), Teste psicológico (Técnica projetiva) e estudos de Reflexão. Já as abordagens quantitativas apareceram em 19,2\% das produções. Os autores trouxeram estudos que utilizaram Qui-quadrado e exato de Fischer, Análise bivariada e de coorte transversal.

No tocante ao papel dos Educadores Sociais destacaram-se temas referentes importância de a formação e capacitação, a fim de, compreender a história dos acolhidos; as práticas de cuidado com os acolhidos, e a qualidade de vida do Educador social. Tais intercessões ofereceram condições para construção da $1^{\mathrm{a}}$ categoria temática - "A formação e identidade do Educador Social e as atribuições no cenário de acolhimento".

E para a $2^{\mathrm{a}}$ categoria versa a partir das temáticas: as vivencias e a imagem do educador no cenário de acolhimento; a imagem que educadores sociais trazem da criança em situação de acolhimento institucional; os vínculos afetivos e o uso da escuta afetiva a percepção de cuidadores de abrigo; nos aspectos de saúde, saúde bucal, maternidades em adolescentes, e a vivencia de violência por adolescentes em situação de rua - "A figura do educador social como um agente de transformação na vida de acolhidos".

\section{Discussão}

Partindo dos dados trabalhados, nesta secção aborda o processo da formação e a identidade do educador social, e a influência na transformação da vida de acolhidos. 


\section{A formação e identidade do Educador Social e as atribuições no cenário de acolhimento}

A partir da implementação do ECA (1990), o processo de institucionalização vem sofrendo mudanças, e um dos focos do reordenamento das unidades de acolhimento institucional está voltado para a formação e capacitação dos ES. Há um consenso entre os pesquisadores quanto à importância da formação e da capacitação como fundamentais para os profissionais das instituições de acolhimento institucional (Avoglia et al., 2012, Barros \& Naiff, 2016, Brito \& Souza, 2011). Os espaços idealizados para a capacitação devem organizar-se de maneira a propiciar trocas de experiências, de cuidado, de ideias, motivação e proteção dos próprios profissionais, e não se limitar apenas à normatização. Nessa lógica, cabe ao espaço ser um lugar de construção metodológica participativa, como troca de auxílio nas atuações cotidianas do ES (Avoglia et al., 2012, Barros \& Naiff, 2016, Lam, 2010). Os profissionais ES reconhecem a importância da capacitação como aprimoramento do processo de trabalho, por lidarem, no cotidiano das instituições, com necessidades específicas dos adolescentes abrigados, provenientes das ruas e que possuem um histórico de exclusão social e familiar que na maioria são jovens que já experienciaram substâncias psicoativas (Penna et al., 2012). As pesquisas apontam que a formulação de cursos de formação, oficinas e reciclagem como ferramenta indispensável para o fortalecimento de discussões sobre suas práticas de cuidado (Avoglia et al.,2012, Penna et al., 2012). A possibilidade de reflexão para os ES deve contribuir para sensibilização em relação ao adolescente, bem como para a construção de ações com qualidade, favorecendo a dimensão educacional a partir das relações cotidianas (Barros \& Naiff, 2016). Nessa perspectiva, os autores defendem a criação de espaços próprios para o educador social lidar com seus sentimentos, angústias, amores e raivas. Um caminho para “cuidar do cuidador” (Magalhães et al., 2011).

A partir desses diálogos, percebe-se que a formação do ES tem sido uma área de frequentes debates ricos e pertinentes entre pesquisadores da profissão, considerando, também, a importância da participação dos próprios educadores na construção das propostas de formação, por meio da reflexão-ação contextualizada no cotidiano do trabalho (Avoglia et al., 2012, Barros \& Naiff, 2016, Brito \& Souza, 2011). Documentos como ECA (1990) e CONANDA (1991) reforçam a ideia de que, para exercer sua função, o ES deve ter capacitação adequada para desempenho do seu papel junto a população de acolhidos. Ainda nesse contexto, o documento "Orientações Técnicas: Serviços de Acolhimento para Crianças e Adolescentes” destaca a importância de investir na capacitação e acompanhamento dos ES. Para tanto, é importante que seja oferecida capacitação inicial de qualidade e formação continuada, especialmente àqueles que têm contato direto com as crianças e adolescentes e suas famílias (Brasil, 2009).

Embora seja indispensável à oferta de supervisão, apoio e capacitação, a regulamentação profissional apresenta fragilidades (Manica, 2017). A formação do Educador Social esbarra em algumas vertentes quanto a regulamentação profissional, através da PLS n. ${ }^{\circ}$ 328/2015, que estabelece as características necessárias ao trabalho, mas centram as atenções no campo de atuação educativa e social (Barros \& Naiff, 2016). Ensaia algo, mesmo que vago, na direção das competências de saber intervir e de mediar as relações socioeducativas na promoção dos sujeitos em vulnerabilidade social. No entanto, essa mesma legislação constitui como um dos critérios à formação mínima do ES seria a graduação. Entretanto, tal exigência não se harmoniza com a Classificação Brasileira de Ocupação (CBO), que estabelece o ensino médio para o desempenho da função de ES. Dessa forma, a delimitação torna-se específica no que se refere às competências e habilidades do ES (Brasil, 2015, Pereira, 2019). Percebe-se uma preocupação de não se subdividirem os serviços entre os profissionais tidos como ES tanto para o nível médio quanto para o graduado (Barros \& Naiff, 2015, Pereira, 2015). Tais processos parecem desalinhados entre si, e podem contribuir para fragilizar a identidade do Educador Social. Ademais, a realidade que compõe grande parte das unidades de acolhimentos, são os educadores sociais, cuidadores, enfim, profissionais com pouca escolaridade, mas que possuem uma representatividade junto à população de acolhidos no país. 
Essa identidade emerge da intercessão de várias práticas e identidades profissionais que têm em comum uma intencionalidade educativa e uma influência a partir dos diversos contextos sociais. Para a construção identitária, os autores referem-se ao papel desenvolvido pelo ES, que ganha sentido no cotidiano das unidades de acolhimento junto a crianças e adolescentes. São profissionais que, por atuarem em escalas extensas de plantão, desempenham a função de cuidado direto, sendo, assim, são considerados elementos fundamentais à interação entre equipe técnica e acolhidos (Avoglia et al., 2012, Brito \& Souza, 2011). A percepção sobre a atuação do ES é identificada pelo desempenho do seu trabalho e pela forte influência sobre o comportamento no processo de socialização primária do acolhido, o que torna de grande importância o seu trabalho nas unidades de acolhimento (Magalhães et al., 2011). Tais aspectos reforçam que a identidade do ES se consolida pela práxis e pela interação com o grupo de adolescentes em situação de acolhimento. A relação estabelecida com os educadores tem sido destacada como fundamental para um atendimento satisfatório (Avoglia et al., 2012, Barros \& Naiff, 2016, Brito \& Souza, 2011).

A caracterização sociodemográfica dos ES encontrada nos estudos aponta que a maioria é pertencente ao sexo feminino, com experiências pessoais referentes à maternidade. São as mulheres, que ao longo da história, ocupam-se do cuidado físico do outro, seja no âmbito doméstico ou profissional. Portanto, concerne a experiências tanto em casa como mães, quanto no campo profissional como educadoras, reforçando, as questões de gênero (Brito \& Souza, 2011, Corrêa et al., 2013).

Geralmente, a faixa etária predominante dos ES varia entre 20 a 56 anos, atuando há mais de dois anos na função. Quanto ao nível de escolaridade, verifica-se a equivalência do ensino médio completo, alguns cursando o ensino superior, o que indica um quadro funcional em processo de qualificação, sendo uma característica positiva para qualidade do atendimento nas unidades de acolhimento institucional (Avoglia et al.,2012, Brito \& Souza, 2011, Corrêa et al., 2013).

Quanto à jornada de trabalho, os ES exercem carga horária semanal, e que devido à caracterização particular de cada unidade de acolhimento, esses profissionais podem aderir regime de plantão. Contudo, observa-se que, mesmo em extensa carga horária, a qualidade de vida dos educadores avaliada na pesquisa foi considerada boa (Brito \& Souza, 2011). Não há dúvidas de que, diante da vulnerabilidade psicossocial dos adolescentes em situação de acolhimento, o ambiente nas unidades pode gerar sobrecarga física e mental aos ES, decorrente das situações advindas dos motivos que levaram esse grupo ao acolhimento (Brito \& Souza, 2011).

\section{A figura do educador social como um agente de transformação na vida de acolhidos}

A figura do ES tem sido essencial para a execução do cuidado não formal e da promoção do autocuidado de criança e do adolescente. Durante o acolhimento institucional o ES é a referência mais próxima de um indivíduo adulto para a população de institucionalizados. São responsáveis pelo cuidar e educar, com gestos de cuidado, diálogo e atenção, contribuindo para a formação de vínculos interpessoais. Desenvolvendo qualidades importantes, tais como zelo, afeto e respeito, a serem impressos no cotidiano com os acolhidos, que tendem a desenvolver essas mesmas qualidades (Avoglia et al.,2012). Segundo Bório et.al. (2020) ao consideramos a formação de vínculo e o apego como indispensáveis para o desenvolvimento infantil, nota-se que no contexto o acolhimento infantil, os vínculos e o apego esses passam a ser exercidos pelos cuidadores ao invés de pelos pais, como ocorre com as crianças que vivem com suas famílias.

A construção de vínculos tem sido um dos pilares que compõe as ações de cuidar e educar cotidianamente para o ES. As ações tanto do educar quanto de cuidar precisam ser harmônicas e concomitantes, sem sobreposição do cuidar sobre o educar, no sentido de assegurar condições favoráveis ao desenvolvimento das condições humanas (Avoglia et al.,2012; Barros \& Naiff, 2015). As práticas do ES, geralmente, buscam auxiliar na construção de novas possibilidades, bem como, o fortalecimento das relações com a população de acolhidos e familiares. Entende-se que as práticas desenvolvidas pelos ES são identificadas como extensivas, propondo a construção de novas perspectivas, com a participação desse profissional ES no 
fortalecimento das relações sociais ou familiares dos adolescentes acolhidos, vistas as diversas situações de fragilidade vividas (Avoglia et al.,2012, Penna et al., 2010, Magalhães et al., 2011, Barros \& Naiff, 2015, Pereira, 2015). Nessa perspectiva o ES tende a mediar e impulsionar a transformação social do grupo de acolhidos. O ES é um agente e mediador da transformação social, ou seja, articulador de um processo coletivo, comprometido com a sociedade, de forma consciente e crítica. Ser agente de transformação é função social do educador. Atuar como tal é conhecer a história de cada buscando contribuir na transformação das realidades encontradas.

Dentre as esferas de transformações na vida dos acolhidos estão as questões que envolve a saúde. Segundo Bispo et al., (2015), o educador deve aproveitar a oportunidade para exercer uma influência positiva sobre as crianças e motivá-las, a fim de desenvolverem bons hábitos de higiene bucal junto ao grupo. Ainda na esfera das questões de saúde, em decorrência de algumas adolescentes ser acolhidas enquanto gestantes ou com seus conceptos. É possível perceber a na literatura a dificuldade que alguns profissionais apresentam para trabalhar as questões que envolvem a sexualidade, a saúde sexual, bem como, a maternidade. Num estudo realizado por Penna et al., (2012), num abrigo que acolhe a mãe adolescente e o filho, como também a adolescente gestante. Os cuidadores acreditam que a maternidade dificulta a reinserção social das adolescentes no mercado de trabalho, diminuindo as possibilidades de uma melhor qualidade de vida após o desabrigamento, e interferindo de forma negativa na situação econômico e financeiro desta adolescente. Apesar dos discursos neste estudo, as autoras perceberam que os profissionais utilizam estratégias para cuidar deste grupo as suas perspectivas pessoais, e que determina a forma de abordagem e manejo junto às adolescentes, conforme suas afinidades, habilidades, posturas e, principalmente, valores pessoais.

Em outra pesquisa realizada por Penna et al., (2010), junto aos cuidadores de um abrigo para adolescentes da rede municipal do Rio de Janeiro, discutem a violência vivida pelas adolescentes. Segundo as autoras, a fala dos cuidadores neste estudo aponta que a problemática da violência vivenciada pelas adolescentes em situação de rua. A violência, apresenta uma gama de inter-relações e concomitância dos vários tipos de violência, configurando a complexa trama da violência que perpassa marcadamente as vidas dessas adolescentes. De tal modo, os cuidadores do abrigo entendem a complexidade e intensidade da violência vivenciada pelas adolescentes em situação de rua, o que mobiliza questões pessoais de cada um.

\section{Considerações Finais}

Neste estudo foi possível compreender que a formação construção da identidade do Educador Social. A pesquisa demonstra que o ES possui o papel de agente transformador na vida dos acolhidos.

O estudo trouxe as fragilidades no que diz respeito aos "processos de formação" do ES. Existem algumas diferenças entre os grupos que discutem não apenas a formação, mas também as habilidades e competências para esta categoria profissional. Ao longo dos anos tais discussões alcançam proporções devido às diversas demandas sociais relacionadas a população de vulneráveis que estes profissionais assistem. Para dos ES, há a necessidade de estabelecer as competências e habilidades para o processo de trabalho. A PLS 328/2019 reforça a necessidade de formação superior para atuação nesta área, porém ainda existe um extenso caminho a ser percorrido, cabendo a reflexão: como será esta nova formação?

Esses profissionais tendem a serem vistos como sujeito ativo na ação de um educar não formal, com responsabilidades para além das rotinas nas instituições. Geralmente, recorrem às suas vivências e experiências adquiridas, seja na esfera familiar ou nos espaços de acolhimento, constituindo um processo formativo que poderá transformará a história de vida do adolescente. Os ES exercem a educação não formal nos espaços de acolhimento institucional, pautada no saber fazer, mesmo que nem sempre obtendo uma qualificação formal para o desempenho de sua práxis. O reconhecimento de que os ES desempenham o papel de agente de transformação, reforça a necessidade de seleção, capacitação e acompanhamento desses profissionais. Nesse sentido, a importância da formação continuada e específica não só nos aspectos relacionados a educação formal, mas também aos aspectos referentes as questões sociais, culturais, de gênero e de saúde, ou seja, questões referentes aos direitos 
sociais, sexuais, de cidadania, é uma ferramenta indispensável para qualidade do cuidado subjetivo desenvolvido no âmbito das unidades de acolhimento.

As considerações advindas dessa análise contribuíram para melhor compreensão do estudo sobre o papel desempenhado pelo educador social no contexto da unidade de acolhimento institucional. Acreditamos que este estudo não se esgota, por entender que o cenário de acolhimento possui diversas lacunas a serem discutidas, a fim de, devolver a sociedade respostas que possam auxiliar no desenvolvimento dos grupos populacionais inseridos neste espaço, bem como, os profissionais que trabalham neste espaço de acolhimento.

\section{Referências}

Avoglia, Hilda Rosa Capelão, Silva, Andreia Maria da, \& Mattos, Pammela Makarowits de. (2012). Educador social: Imagem e relações com crianças em situação de acolhimento institucional. Revista Mal-estar e Subjetividade, 12(1-2), 265-292.

Bardin, L. Análise de conteúdo. (2016). São Paulo: Editora: Almedina.

Barros, Nilma Soares, \& Naiff, Luciene Alves Miguez. (2015). Capacitação para educadores de abrigo de crianças e adolescentes: identificando representações sociais. Estudos e Pesquisas em Psicologia, 15(1), 240-259

Bassoli, Eiza Nádila, Figueiredo,Vanessa Cartherina Neumann. (2020). Desafios do trabalho de educadoras sociais em casas de acolhimento. Revista IberoAmericana de Estudos em Educação, Araraquara, 15(n. esp3), 2396-2410.

Bispo, J.R. et al. (2015). Conhecimento dos educadores do abrigo Tereza de Jesus sobre saúde bucal infantil. Revista de Odontologia da Universidade da Cidade de São Paulo. São Paulo, 27(3), 202-209.

Bório, T. C., Gabatz, R. I. B., Milbrath, Schwartz, V. M., Eda, Vaz, Jéssica Cardoso (2020). Institucionalização infantil: revisão acerca da interação dos cuidadores com a criança. Revista Brasileira de Revisão de Saúde, 3(1), jan./fev. Curitiba.

Botelho, L. L. R., Cunha, C. C. de A., \& Macedo, M. (2011). O método da revisão integrativa nos estudos organizacionais. Gestão e Sociedade, 5(11), 121136. https://doi.org/10.21171/ges.v5i11.1220.

Brito, Cristiane Vinholi de, \& Souza, José Carlos. (2011). Qualidade de vida dos educadores sociais em abrigos de proteção a crianças e adolescentes. Psicologia: teoria e prática, 13(1), 89-100.

Carvalho, R. M. C., Cardoso, E. L. S, Ferreira, B. M. S, \& Ferreira, R. K. G. (2020). COVID-19 na pediatria: uma revisão integrativa. Research, Society and Development, 9 (9), e322997140.

Cavalcante, Lília Iêda Chaves, \& Corrêa, Laiane Da Silva. (2012). Perfil e trajetória de educadores em instituição de acolhimento infantil. Cadernos de Pesquisa, 42(146), 494-517.

Cintra, Ana Lúcia, \& Souza, Mériti de. (2010). Institucionalização de crianças: leituras sobre a produção da exclusão infantil, da instituição de acolhimento e da prática de atendimento. Revista Mal-estar e Subjetividade, 10(3), 809-833

Corrêa, Laiane da Silva, \& Cavalcante, Lília Iêda Chaves. (2013). Educadores de abrigo: concepções sobre desenvolvimento e práticas de cuidado em situação de brincadeira. Journal of Human Growth and Development, 23(3), 309-317.

Cunha, R.C. (2011). O educador social e sua inserção nas políticas públicas: imprescindibilidade ou rearranjo do capital? Revista Espaço de Diálogo e Desconexão. Araraquara. 3(2),4438

Elage, B. et.al. (2011). Perspectivas: formação de profissionais em serviço de acolhimento. 1 Ed. Instituto Fazendo História: São Paulo.

Galvão, Taís Freire, \& Pereira, Mauricio Gomes. (2014). Revisões sistemáticas da literatura: passos para sua elaboração. Epidemiologia e Serviços de Saúde, 23(1), 183-184.

Garcia Penna, Lúcia Helena, dos Santos Fernandes, Ravini, Rosane Guedes, Cláudia, Pérsia Paulo dos Santos, Úrsula, da Silva Fernandes, Gleice, \& Yuan Soares, Beatriz. (2012). Concepção de profissionais de unidades de acolhimento sobre a maternidade em adolescentes abrigadas. Rev Rene, 13(1),44-52. ISSN: $1517-3852$

Iannelli, Andrea M., Assis, Simone Gonçalves, \& Pinto, Liana Wernersbach. (2015). Reintegração familiar de crianças e adolescentes em acolhimento institucional em municípios brasileiros de diferentes portes populacionais. Ciência \& Saúde Coletiva, 20(1), 39-48.

Ito, S. I. \& Azevêdo, A. V. dos S. (2021). Educadores Sociais em Abrigos Destinados a Crianças e Adolescentes: revisão sistemática. Contextos Clínicos, $14(1)$, jan./abr.

Lam, Carla. (2010). Holding e rêverie: postura do coordenador de grupo de reflexão com educadoras em um abrigo. Vínculo, 7(1), 33-43.

Lei $\mathrm{n}^{\circ}$ 8.242, de 12 de outubro de 1991 (1991). Conanda. Dispõe sobre o Conselho Nacional dos Direitos da Criança e do Adolescente. Brasília, DF. https://www.gov.br/participamaisbrasil/conanda 
Lei $\mathrm{n}^{\circ}$ 8.069, de 13 de julho de 1990. (1990). Dispõe sobre o Estatuto da Criança e do Adolescente e dá outras providências. Brasília, DF. http://www.planalto.gov.br/ccivil_03/leis/L8069.

Lei $\mathrm{n}^{\circ} 12.010$ de 03 de agosto de 2009. (2009). Dispõe sobre adoção. Casa Civil, Brasília.http://www.planalto.gov.br/ccivil_03/_Ato20072010/2009/Lei/L12010.htm

Liberati, A., Altman, D. G., Tetzlaff, J., Mulrow, C., Gøtzsche, P. C., Ioannidis, J. P., Clarke, M., Devereaux, P. J., Kleijnen, J., \& Moher, D. (2009). The PRISMA statement for reporting systematic reviews and meta-analyses of studies that evaluate health care interventions: explanation and elaboration. PLoSMedicine, 6(7), e1000100.

Lucca, A., Griecco, A., Franco, F., \& Prado, R. (2018). Construção da identidade do educador social. Diálogos Interdisciplinares, 7(4), 45-53. https://revistas.brazcubas.br/index.php/dialogos/article/view/587

Magalhães, C.M.C, Costa, L.N. \& Cavalcante, L.I.C. (2011). Percepção de educadores de abrigo: o seu trabalho e a criança institucionalizada. Journal of Human Growth and Development. São Paulo, 21(3), 818-831

Manica, L. E. A educação profissional formal e não formal das pessoas com deficiência no Brasil. Revista Ibero-Americana de Estudos em Educação, Araraquara, 12(4), 998-2023, out./dez. 2017.

Ministério do Desenvolvimento Social Brasil (2009). Secretaria Especial dos Direitos Humanos. SEDH. Conselho Nacional dos Direitos da Criança e do Adolescente. Orientações Técnicas: Serviços de Acolhimento para Crianças e Adolescentes. Brasília, DF.

Ministério do Desenvolvimento Social. Secretaria Especial dos Direitos Humanos. SEDH. (2009). Conselho Nacional dos Direitos da Criança e do Adolescente Orientações Técnicas: Serviços de Acolhimento para Crianças e Adolescentes. Brasília, DF. https://www.mds.gov.br/webarquivos/publicacao/assistencia_social/Cadernos/orientacoes-tecnicas-servicos-de-alcolhimento.pdf.

Ministério do Desenvolvimento Social Brasil (2018). Censo SUAS 2017: análise dos componentes sistêmicos da política nacional de assistência social. Secretaria de Avaliação e Gestão da Informação; Secretaria Nacional de Assistência Social, Brasília, DF.

Ministério do Desenvolvimento Social e Combate à Fome. (2006). Conselho Nacional de Assistência Social. Norma operacional básica de recursos humanos do suas NOB-Rh/SUAS. https://www.mds.gov.br/webarquivos/publicacao/assistencia_social/Normativas/NOBRH_SUAS_Anotada_Comentada.pdf

Ministério do Trabalho e Previdência. (2021). Classificação Brasileira de Ocupações - CBO. http://www.mtecbo.gov.br/cbosite/pages/home.jsf

Moreira, T. A. S., \& Paiva, I. L. de. (2015). A atuação do psicólogo nos Serviços de Acolhimento Institucional de crianças e adolescentes. Psicologia Em Estudo, 20(3), 507-517.

Nörnberg, M., \& Menezes M. (2012). Práticas de Acolhimento em Abrigo Infanto-Juvenil. Revista Contexto \& Educação, 26(85), 177-201.

Oliveira, G. T. S. (2017). Do lar doméstico ao lar institucional: a percepção sobre o trabalho na ótica das cuidadoras sociais de casas e lares de Curitiba e região metropolitana. 156 f. Dissertação (Mestrado em Tecnologia e Sociedade) - Universidade Tecnológica Federal do Paraná, Curitiba.

Penna, L., Carinhanha, J., Ribeiro, L., Graça, H., \& Marques, C. (2017). Perfil sociodemográfico da adolescente em situação de rua: análise das condições socioculturais [Socio-demographic profile of female adolescents in a street situation: analysis of sociocultural conditions]. Revista Enfermagem UERJ, 25, e29603.

Pereira, A. S., Shitsuka, D. M., Parreira, F. J. \& Sitsuka, R. (2018). Metodologia da pesquisa científica. UFSM.

Pereira, A. (2019). O educador social e suas competências de atuação profissional: um trabalhador da Educação pela LDBEN n. ${ }^{\circ}$ 9.394/96?. Debates em Educação, 11(23), 311-332.

Paiva, J., Souza, C. R. T. de, Natali, P. M., Müller, V. R., \& Bauli, R. A. (2014). A atuação profissional e formação do educador social no Brasil: uma roda da conversa. Interfaces Científicas - Educação, 3(1), 77-88.

Rodrigues, J. L. dos S., Santos, L. M.M. dos \& Lima, A.F. de (2021). Mudar, pensar em mudar, continuar mudando: Narrativas das metamorfoses de uma Adolescência em abrigamento. Psicologia \& Sociedade - v. 33 e217494.

Rodrigues, R., Penna, L., Ribeiro, L., Paes, M., \& Guedes, C. (2015). Sexualidade das adolescentes em situação de acolhimento: contexto de vulnerabilidade para DST [Sexuality of adolescent girls in foster care: context of vulnerability to STD]. Revista Enfermagem UERJ, 23(4), 507-512.

Senado Federal do Brasil (2015). Projeto de Lei no 328/2015. Dispõe sobre a regulamentação da profissão de educadora e educador social e dá outras providências. Apresentado pelo senador Temário Mota em 2 de junho de 2015. Brasília, DF, 2015.

Souza, C.R.T de; Paiva, J.; Natali, P.M.; Bauli, R.A. \& Müller, V.R. A atuação profissional e formação do educador social no Brasil: Uma roda da conversa. Interfaces Científicas-Educação. Aracaju, 3(1), 77-88, out. 2014.

Trilla, J. (2003). O universo da Educação Social. In: Romans, M.; Petrus, A. Trilla, J. Profissão: Educador Social. Artmed.

Trivellato, Aline Jacob, Carvalho, Cíntia, \& Vectore, Celia. (2013). Escuta afetiva: possibilidades de uso em contextos de acolhimento infantil. Psicologia Escolar e Educacional, 17 (2), 299-307. 